\title{
Minimally invasive direct coronary artery bypass for left anterior descending artery revascularization - analysis of 300 cases
}

\author{
Lufeng Zhang, Zhongqi Cui, Zhiming Song, Hang Yang, Yuanhao Fu, Yichen Gong, Yunpeng Ling \\ Third Hospital of Peking University, Beijing, China
}

Videosurgery Miniinv 2015; 10 (4): 548-554

DOI: $10.5114 /$ wiitm.2015.55842

\begin{abstract}
Introduction: Minimally invasive direct coronary artery bypass (MIDCAB) grafting is performed via small, left anterolateral thoracotomy. The left internal mammary artery was grafted to the left anterior descending (LAD) artery in 300 consecutive patients.

Aim: In-hospital results were evaluated and compared with the conventional, off-pump coronary artery bypass graft procedure.

Material and methods: One hundred and sixty-three (54.33\%) of 300 patients underwent staged hybrid coronary revascularization, 93 (31\%) were treated for a single $L A D$ lesion, and 44 (14.67\%) were treated for multi-vessel disease with reasonably incomplete revascularization. Major in-hospital cardiac adverse events and postoperative data were compared between groups.

Results: Preoperative data were similar between groups. However, the difference in left ventricular ejection fraction ( $p<0.001)$ was significant. No conversions to sternotomy occurred during the primary MIDCAB procedures. Shorter operation time $(p<0.001)$, shorter postoperative mechanical ventilation time $(p<0.001)$, shorter intensive care unit stay $(p<0.001)$, and less red blood cell transfusion $(p<0.001)$ were noted in the MIDCAB group. Postoperative coronary angiography conducted in the MIDCAB group within one week after the operation showed that 2 of 163 patients developed graft occlusion. These patients underwent conventional CABG and recovered well. No significant differences were observed in postoperative MI, delayed wound healing or 30-day in-hospital mortality between the two groups.

Conclusions: The use of a chest wall lifting system and modified stabilizer makes the MIDCAB procedure safer and easier. The MIDCAB procedure is demonstrated to be a feasible and minimally invasive alternative for patients with coronary artery disease involving $L A D$ lesions.
\end{abstract}

Key words: coronary artery bypass grafts surgery, off-pump surgery, minimally invasive surgery.

\section{Introduction}

Coronary artery bypass grafting (CABG) is an evidence-based, effective treatment method for patients with coronary artery disease (CAD). The most advantageous outcome of CABG is the graft of the left internal mammary artery (LIMA) to the left anterior descending (LAD) artery. This specific graft is associated with reduced risk of death, myocardial infarction, and recurrent angina, and this method has been proven to be strongly associated with excellent long-term patency and survival rates [1, 2]. Despite rapid improvements in trans-catheter techniques and the deployment of drug-eluting stents, a broad scientific consensus has confirmed the unparalleled patency rate of the LIMA when grafted to

\section{Address for correspondence}

Yunpeng Ling MD, Third Hospital of Peking University, 49 North Garden Rd, 100191 Beijing, China, phone: +86 1082266951 ,

e-mail: micsling@163.com 
the LAD artery [2]. Direct LIMA to LAD artery grafting via a small, left anterior thoracotomy was first introduced into clinical practice by Dr. Kolessov [3]. This approach was not initially widely adopted due to the technical complexity of LIMA harvesting and the poor quality of the anastomoses performed on the beating heart. Recently, we introduced a chest wall lifting system for direct LIMA harvesting and a modified stabilizer for LAD anastomoses into this kind of surgery. We have performed this procedure in 300 cases.

\section{Aim}

This study was designed to compare the minimally invasive direct coronary artery bypass (MIDCAB) procedure with the conventional off-pump coronary artery bypass (OPCAB) procedure. The safety of the MIDCAB procedure for the treatment of $C A D$ involving the $L A D$ artery was also assessed.

\section{Material and methods}

From May, 2012 to May, 2015, 300 consecutive patients with CAD involving the LAD artery without $(93 / 300,31 \%)$ or with $(207 / 300,69 \%)$ a significant lesion in at least 1 (in addition to the LAD) major epicardial vessel underwent the MIDCAB procedure for LIMA-LAD artery grafting. Among 207 patients with multi-vessel disease, 163 (54.33\%) were part of a staged hybrid revascularization. The rest of 44 (16.47\%) patients with multi-vessel disease were selected for isolated LIMA-LAD grafting, and the non$L A D$ vessels were not treated due to increased age, preoperative comorbidities, small epicardial vessel

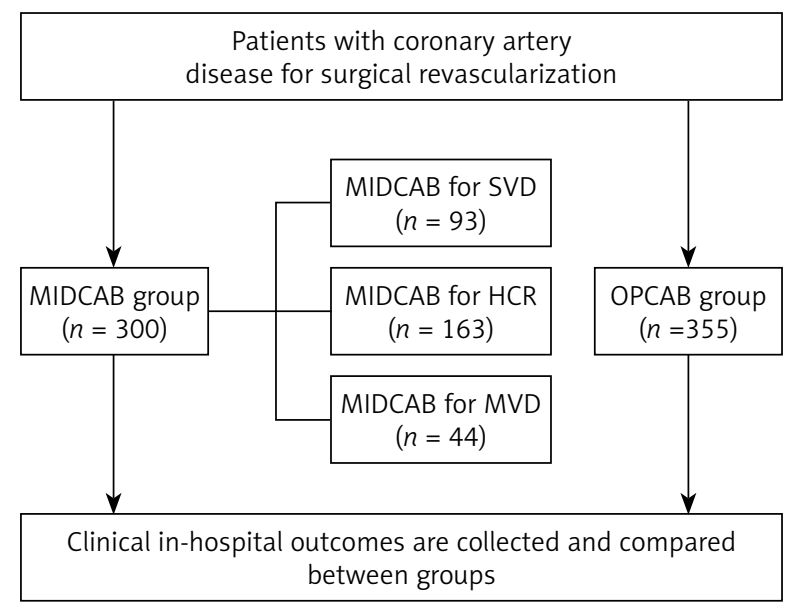

Figure 1. Design of study flow chart
( $<1 \mathrm{~mm}$ ) diameter that were not able to be revascularized either by CABG or percutaneous coronary intervention ( $\mathrm{PCl})$, or patient preference. In this study, patients who underwent the MIDCAB procedure were matched with 355 patients who underwent off-pump, multi-vessel bypass grafting via sternotomy (at the same medical center during the same period). The perioperative data were analyzed and compared between groups. This study was approved by the Institutional Review Board of Peking University before any study-related procedure was performed. The study flow chart is shown in Figure 1.

Surgical procedure: In the MIDCAB group, patients were anesthetized and intubated with double-lumen endotracheal tubes. Single-lung ventilation was employed to facilitate exposure for LIMA harvesting. Patients were positioned supine with a $30^{\circ}$ rotated decubitus towards the right by means of a rolled towel. External pads for emergency defibrillation were placed on the right anterior and left posterior sides of the chest wall. A small, left anterior thoracotomy incision $(5-7 \mathrm{~cm})$ was performed in the $4^{\text {th }}$ or $5^{\text {th }}$ intercostal space, according to the preoperative chest X-ray and coronary angiography.

The hook length required for chest wall lifting was determined by measuring the distance between the incision and the $1^{\text {st }}$ rib. After proper retractor positioning for LIMA harvesting, the direction of the hook was adjusted according to the shape of the chest wall (Photo 1). Importantly, the chest wall should be lifted gradually and the ribs should be spread slowly to avoid rupture of the sterno-costal joint or costal cartilage, which could prevent postoperative chest pain. Typically, LIMA harvesting is

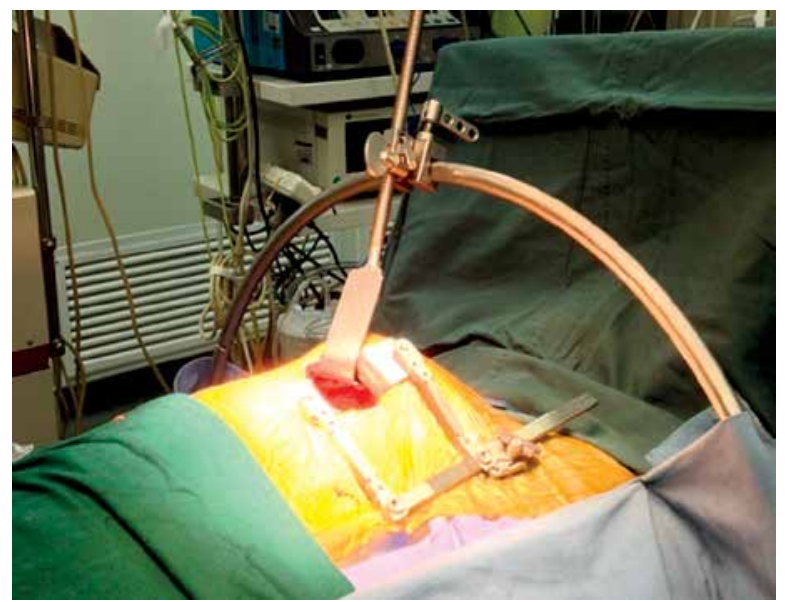

Photo 1. Chest wall lifting system 


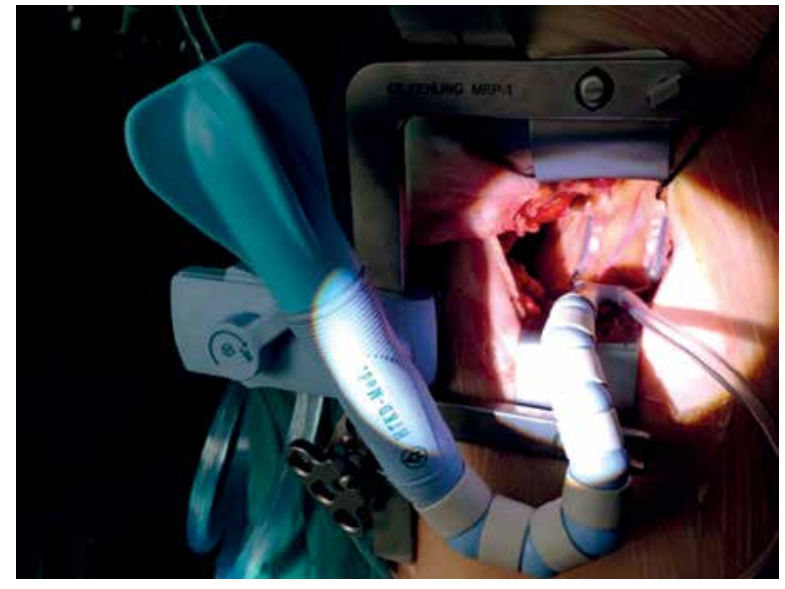

Photo 2. Minimally invasive stabilizer

initiated from superior to inferior because there is no transthoracic muscle at the superior border. Fat tissue was removed for identification of the LIMA, which was harvested with a pedicle. Two branches of the LIMA (up to the pericardium) were routinely dissected and clipped. The LIMA was harvested superior to the $1^{\text {st }}$ rib (close to left subclavian vein) and inferior to the upper portion of the $5^{\text {th }}$ or $6^{\text {th }}$ costal cartilage. Clips were used to dissect branches of the LIMA. To achieve a successful MIDCAB procedure, the length of the LIMA harvested should be maximized.

After LIMA preparation and dilation with papaverine solution, blood flow from the LIMA was confirmed. A pericardial incision was performed to expose and stabilize the LAD. The LAD artery is typically located on the apical, right side and travels caudally, parallel to the sternum. A well-developed diagonal artery, which parallels the skin incision, can be mistaken as the LAD artery. The anatomical landmarks for the intra-myocardial LAD artery include the left margin of the pulmonary artery and the great cardiac vein, which travels parallel to the LAD artery, and the origin of the diagonal arteries.

The authors developed a specialized, suctionbased stabilizer for the MIDCAB procedure (HT-KD, China). The stabilizer pods for target vessel stabilization were designed in an "L" (rather than "- " style) to facilitate manipulations in deep, small incisions. Moreover, the stabilizer could be mounted onto the arm of a minimally invasive retractor (Photo 2). Hemodynamic instability was minimized by applying two stabilizer pods with suction to the epicardium around the target vessel. A shunt was routinely used after coronary arteriotomy instead of target vessel occlusion. Maintaining myocardial perfusion during the anastomosis is crucial during the MIDCAB procedure, since ventricular tachycardia or hemodynamic instability caused by myocardial ischemia can lead to an emergency conversion to sternotomy. A continuous running suture (8-0) technique was used to perform the coronary anastomosis. Precision anastomosis of the LAD artery to the LIMA is important for both short- and long-term graft patency.

In the conventional OPCAB group, coronary revascularization was performed in 355 patients via sternotomy using pedicled LIMAs for LAD artery grafting. The saphenous vein or radial artery was used for non-LAD vessel grafting. A suction-based epicardial stabilizer and intracoronary shunt were routinely used. Proximal anastomoses were performed with a side clamp.

Patients were postoperatively transferred to the intensive care unit (ICU) and supported with mechanical ventilation. Perioperative data were collected and analyzed for both groups. A total of 162 patients in the staged hybrid coronary revascularization subgroup underwent $P C I$ for non-LAD vessels, and LIMA to LAD graft patency was assessed at the same time.

\section{Statistical anlaysis}

Data collection and statistics: Continuous variables are presented as means \pm SD. Categorical variables are presented as percentages. A $p$-value $<0.01$ was considered significant. Statistical tests were performed with SPSS version 19.0.

\section{Results}

A total of 300 patients underwent MIDCAB while 355 patients underwent OPCAB from May, 2012 to May, 2015 for the treatment of CAD involving LAD artery lesions. Perioperative data were compared between the OPCAB and MIDCAB groups. There were no significant preoperative differences, except for left ventricular ejection fraction (EF). Preoperative data are shown in Table I.

In the MIDCAB group, with the chest wall lifting system and direct vision, the LIMA harvesting time was 25-105 min (41 $\pm 21 \mathrm{~min})$. LIMA injury occurred in 1 of 300 patients $(1 / 300,0.33 \%)$. A long segment of the saphenous vein was anastomosed to the left subclavian artery and passed through the $1^{\text {st }}$ inter- 
Table I. Baseline characteristics of patients according to study groups

\begin{tabular}{|lccc|}
\hline Parameter & $\begin{array}{c}\text { MIDCAB group } \\
(n=300)\end{array}$ & $\begin{array}{c}\text { OPCAB group } \\
(n=355)\end{array}$ & Value of $p$ \\
\hline Age [year] & $62.6 \pm 10.2$ & $60.4 \pm 8.8$ & 0.431 \\
\hline Gender (female \%) & 22.90 & 25.91 & 0.371 \\
\hline Height [cm] & $164.1 \pm 24.5$ & $166.6 \pm 7.5$ & 0.078 \\
\hline Weight [kg] & $70 \pm 10.2$ & $70.69 \pm 10.72$ & 0.73 \\
\hline NYHA classification & $1.68 \pm 0.69$ & $1.79 \pm 0.57$ & 0.034 \\
\hline Diabetes mellitus (\%) & 34.30 & 26.20 & 0.08 \\
\hline Hypertension (\%) & 52.70 & 50.14 & 0.519 \\
\hline Tobacco use (\%) & 48.80 & 49.01 & 0.819 \\
\hline Cerebrovascular stenosis (\%) & 12.90 & 12.96 & 0.856 \\
\hline Creatinine level & $80.39 \pm 106.19$ & $69.61 \pm 16.55$ & 0.026 \\
\hline Left main artery lesion (\%) & 14.40 & 13.52 & 0.749 \\
\hline Prior myocardial infarction (\%) & 28.40 & 20.56 & 0.034 \\
\hline Ejection fraction (\%) & $63.36 \pm 9.48$ & $58.92 \pm 15.67$ & $<0.001^{*}$ \\
\hline
\end{tabular}

Data presented as mean $\pm S D$ or $\%$. ${ }^{*} P<0.01$. SD - standardized difference in mean, MIDCAB - minimally invasive direct coronary artery bypass, OPCAB - offpump coronary artery bypass, NYHA - New York Heart Association, LVEDD - left ventricular end-diastolic diameter.

costal space. This vein graft was anastomosed to the LAD artery in a manner similar to LIMA-LAD artery grafting. The length of the LIMA was sufficient for most LAD anastomoses. However, in 3 cases (3/300, $1 \%)$, a short segment of the saphenous vein was used for LIMA extension due to dissection of the distal end of the LIMA or an anastomosis site on the distal portion of the LAD artery. The average graft number for the OPCAB group was $2.76 \pm 0.6$, including LIMA-LAD grafting and saphenous vein or radial artery to non-LAD artery grafting.

We found significant differences between the MIDCAB and OPCAB groups regarding operation time $(152.0 \pm 43.5$ vs. $263.2 \pm 52.4 \mathrm{~min}, p<0.001)$, postoperative mean ventilation time for respiration support (9.26 \pm 7.76 vs. $24.85 \pm 39.69$ h, $p<0.001)$, ICU length of stay $(24.27 \pm 17.85$ vs. $59.13 \pm 60.39 \mathrm{~h}$, $p<0.001)$ and total red blood cell transfusion (0.79 \pm 1.58 vs. $3.2 \pm 5.01$ units, $p<0.001)$. In the MIDCAB group, 2 patients underwent re-exploration (via thoracotomy) to clip a bleeding branch of the LIMA. Two patients with multi-vessel vessel lesions were staged for hybrid revascularization and were diagnosed with postoperative myocardium infarction based on the symptoms, electrocardiograms and myocardial injury biomarkers. One patient showed a new Q-wave in the inferior wall without hemodynamic instability and was treated with $\mathrm{PCI}$. An occlusion of a preoperatively placed stenosis in the right coronary artery was identified and successfully revascularized with a drug-eluting stent (DES). The patient recovered well and was discharged after $\mathrm{PCl}$. As for another patient, conventional CABG with CPB was performed after ST segment elevation in ECG, which revealed anterior and lateral wall infarctions. He died of heart failure. The perioperative mortality rate was $0.33 \%$ (1/300). Postoperative data are shown in Table II.

A total of 162 (of 300) patients underwent staged hybrid coronary revascularization, and 162 (of 300, 54\%) underwent coronary angiography within one week after MIDCAB. The LIMA graft occlusion occurred in 2 patients (2/162, 1.23\%). Offpump CABG was performed via sternotomy (instead of hybrid revascularization) for both of them. LIMA grafts were patented during reoperation, and blood flow occlusion appeared due to stenosis of the native coronary artery. No conversions to sternotomies occurred during the primary MIDCAB procedure. No postoperative strokes occurred in either group. One patient in the MIDCAB group with preoperative renal insufficiency underwent hemofiltration and recovered well. Delayed wound healing without infection 
Table II. In-hospital outcome of patients according to the study groups

\begin{tabular}{|c|c|c|c|}
\hline Parameter & $\begin{array}{l}\text { MIDCAB group } \\
\quad(n=300)\end{array}$ & $\begin{array}{l}\text { OPCAB group } \\
(n=355)\end{array}$ & Value of $p$ \\
\hline Type of operation: & MIDCAB & OPCAB & \\
\hline Isolate MIDCAB for SVD & \multicolumn{2}{|c|}{$93 / 300,31 \%$} & \\
\hline Staged hybrid operation & \multicolumn{2}{|c|}{$163 / 300,54.33 \%$} & \\
\hline Isolate MIDCAB for MVD & \multicolumn{2}{|c|}{$44 / 300,14.67 \%$} & \\
\hline LIMA harvesting time [min] & \multicolumn{2}{|c|}{$25-105(41 \pm 21)$} & \\
\hline Operation time [min] & $152.0 \pm 43.5$ & $263.2 \pm 52.4$ & $<0.001^{*}$ \\
\hline Postoperative ventilation [h] & $9.27 \pm 5.14$ & $24.92 \pm 37.87$ & $<0.001^{*}$ \\
\hline ICU length of stay [h] & $24.27 \pm 17.25$ & $59.13 \pm 60.39$ & $<0.001^{*}$ \\
\hline Total transfusion of RBC [U] & $0.79 \pm 1.58$ & $3.26 \pm 5.02$ & $<0.001^{*}$ \\
\hline Re-exploration for bleeding & $2 / 300,0.67 \%$ & $3 / 355,0.85 \%$ & 0.794 \\
\hline Postoperative MI & $2 / 300,0.67 \%$ & $2 / 355,0.56 \%$ & 0.124 \\
\hline Wound infection & $1 / 300,0.33 \%$ & $3 / 355,0.85 \%$ & 0.403 \\
\hline 30-day mortality & $1 / 300,0.33 \%$ & $3 / 355,0.85 \%$ & 0.403 \\
\hline
\end{tabular}

Data presented as mean $\pm S D$ or $\%$. ${ }^{*} P<0.01$. SD - standardized difference in mean, MIDCAB - minimally invasive direct coronary artery bypass, OPCAB - offpump coronary artery bypass, LIMA - left internal mammary artery, ICU - intensive care unit, RBC - red blood cell, MI - myocardium infarction.

occurred in 1 patient in the MIDCAB group and in 3 patients in the OPCAB group $(p=0.403)$.

\section{Discussion}

Coronary artery disease involving the LAD artery should be treated via LIMA grafting, which is associated with excellent long-term patency and survival rates. Direct LIMA harvesting via a left anterior thoracotomy is a minimally invasive alternative to the conventional procedure, and the learning curve is minimal compared to the endoscopic procedure. A robotic LIMA harvesting technique is easier than direct harvesting and prevents severe postoperative chest pain. However, the robotic technique increases the medical cost and thus limits its application in developing countries.

We used a chest wall lifting system and a minimally invasive thoracotomy retractor for direct LIMA harvesting. Commercially available retractors were used to facilitate exposure. Our FEHLING retractor system (FEHLING, Germany) provided excellent exposure. This system could reduce the potential injury to the LIMA and is the default system in our center. The mean time for LIMA harvesting was 41 \pm 11 min, which was shorter than thoracoscopic or robotic LIMA harvesting [4]. One issue regarding non-endoscopic or non-robotic MIDCAB LIMA harvesting is whether the MIDCAB procedure provides an adequate graft length. In our study, 4 of 300 patients required partial (3) or total (1) elongation of the LIMA with a vein graft, which was acceptable. Although we did not perform follow-up coronary angiography for these 3 patients whose LIMA was elongated with a short segment of vein graft, theoretically the patency of the graft was lower than that of the LIMA-LAD graft and similar to a vein graft.

The deep surgical field and small working space necessitated modified stabilizer pods (“L” style) for the MIDCAB procedure. The vacuum suction stabilizer created less compression on the ventricle, which induced slight hemodynamic instability. A shunt was used to perform a precise anastomosis. The shunt preserved coronary flow [5] and reduced pressure for the operator.

The MIDCAB procedure was intended to treat isolated $L A D$ artery disease. With the development of the $\mathrm{PCI}$ technique, only LAD arteries with a bifurcation of the lesion, long and defuse stenosis, calcification or chronic total occlusion should be treated with surgical revascularization. Compared to the $\mathrm{PCI}$ technique, surgical LIMA-LAD grafting results in 
fewer revascularizations and better survival rates according to a 10-year follow-up study with minimal invasiveness [6].

Most of the patients in the MIDCAB group (54.33\%), who are typically assigned to the conventional CABG procedure, were treated with hybrid revascularization for multi-vessel disease. During the application of staged hybrid revascularization, cardiac surgeons and interventionists discussed the anatomy of the coronary lesions and developed a strategy for staged revascularization. Generally, MIDCAB is performed first and then $\mathrm{PCl}$. The $\mathrm{PCl}$ is performed first when a non-LAD artery is the culprit vessel and a reduction of blood flow during $L A D$ anastomosis could lead to hemodynamic instability. A left main lesion is not a contraindication for HCR, because LIMA to LAD grafting protects blood flow for left circumflex artery (LCX) stenting [7]. In this study, we did not compare HCR group patients with the OPCAB group. A specific study focus on comparison of these two groups is ongoing. We will present data to compare results between MIDCAB + PCI (HCR) and the OPCAB procedure as treatment for multi-vessel disease.

A relatively small population (14.67) of patients with multi-vessel disease was referred to our surgical department. These patients underwent incomplete revascularization during isolated LIMA to LAD grafting due to age, multiple preoperative comorbidities, patient preference, or small, non-LAD target vessels $(<1 \mathrm{~mm})$ that could not be revascularized by $\mathrm{PCI}$ or $C A B G$. The $L A D$ artery provides approximately $60 \%$ of the myocardium blood flow. During LIMA to LAD artery bypass, incomplete revascularization of the circumflex or right coronary artery did not adversely affect early or long-term survival in patients with multi-vessel CAD. However, it is notable that in patients with 1 poor target vessel, incomplete revascularization is a good therapeutic option, and the benefit of complete revascularization should be balanced against the potential risks [8]. In our study, the early operative results were encouraging.

Patients with impaired ejection fraction (EF < 50\%) numbered $49 / 355$ (13.8\%) in the OPCAB group and $35 / 300(11.7 \%)$ in the MIDCAB group. For multi-vessel diseased patients with impaired EF, our concern focuses more on complete revascularization. We do not have a preference for a certain technique although the HCR group does show a shorter period of recovery postoperatively.
In the MIDCAB procedure for single vessel disease and the OPCAB group, we use oral aspirin preand postoperatively. In the MIDCAB-first staged HCR group, aspirin was used before the MIDCAB procedure. Oral aspirin and clopidogrel were started after MIDCAB for a few days before the $\mathrm{PCI}$ procedure. After $\mathrm{PCl}$, oral Plavix for 1 year and aspirin for lifetime were the standard anticoagulation protocol. In the first staged HCR group, oral aspirin and clopidogrel were used pre- and post-operatively.

The key points for successful MIDCAB procedures are long, patent LIMAs, local myocardium stabilization and precise anastomosis. In our study, MIDCAB could be employed as a treatment for isolated LAD lesions, as part of staged hybrid revascularization procedures, or as reasonable incomplete coronary revascularizations for patients who are too sick to undergo conventional CABG for complete coronary revascularization.

Overall, the MIDCAB procedure is comparable to the conventional OPCAB procedure and is associated with shorter postoperative mechanical ventilation time, shorter ICU length of stay and fewer blood transfusions.

\section{Conclusions}

Our results show that the use of a chest wall lifting system and modified stabilizer makes the MIDCAB procedure safer and easier. The MIDCAB procedure is demonstrated to be a feasible and minimally invasive alternative for patients CAD involving LAD lesions.

\section{Conflict of interest}

The authors declare no conflict of interest.

\section{References}

1. Loop FD, Lytle BW, Cosgrove DM, et al. Influence of the internal-mammary-artery graft on 10-year survival and other cardiac events. N Engl J Med 1986; 314: 1-6.

2. Jeremiah U, Panch J, Branavan U, et al. Barriers to the universal adoption of bilateral internal mammary artery grafting. Int J Surg 2015; 16: 179-82.

3. Kolessov VI. Mammary artery-coronary anastomosis as method of treatment for angina pectoris. J Thorac Cardiovasc Surg 1967; 54: 535-44.

4. Olivier J, Fabrice W, Thomas S, et al. Is there an optimal minimally invasive technique for left anterior descending coronary artery bypass? J Cardiothorac Surg 2011, 6: 37.

5. Yoshiyuki T, Kazuyoshi T, Wataru K, et al. Clinical validation of coronary artery flow through an intracoronary shunt during 
off-pump coronary artery bypass grafting. J Thorac Cardiovasc Surg 2014; 147: 259-63.

6. Umberto B, Shahzad GR, Rafik FS, et al. Minimally invasive direct coronary artery bypass improves late survival compared with drug-eluting stents in isolated proximal left anterior descending artery disease: a 10-year follow-up, single-center propensity score analysis. J Thorac Cardiovasc Surg 2014; 148 : 1316-22.

7. Michael EH, Tanveer R, Thomas AV, et al. Hybrid coronary revascularization versus off-pump coronary artery bypass for the treatment of left main coronary stenosis. Ann Thorac Surg 2011; 92: 2155-60.

8. Ardawan JR, Thomas W, Volkmar F, et al. Does reasonable incomplete surgical revascularization affect early or long-term survival in patients with multi-vessel coronary artery disease receiving left internal mammary artery bypass to left anterior descending artery? Circulation 2009; 120 (Suppl. 1): S70-7.

Received: 6.06.2015, accepted: 20.10.2015. 